\title{
Impact of Check-In Data on Urban Vitality in the Macao Peninsula
}

\author{
Chen Pan, ${ }^{1}$ Junling Zhou, ${ }^{2}$ and Xiaohua Huang $\mathbb{D}^{3}$ \\ ${ }^{1}$ Faculty of Innovation and Design, City University of Macau, Macau 99078, China \\ ${ }^{2}$ Guangdong Polytechnic Normal University, Guangzhou, Guangdong Province 510665, China \\ ${ }^{3}$ School of Design, NingboTech University, Ningbo 315100, China \\ Correspondence should be addressed to Xiaohua Huang; hxh@nit.zju.edu.cn
}

Received 7 July 2021; Revised 24 July 2021; Accepted 14 August 2021; Published 7 September 2021

Academic Editor: Chenxi Huang

Copyright $\odot 2021$ Chen Pan et al. This is an open access article distributed under the Creative Commons Attribution License, which permits unrestricted use, distribution, and reproduction in any medium, provided the original work is properly cited.

\begin{abstract}
High activity is an important manifestation of the stable development of urban social economy. Quantitative research on urban development based on the geographical label perception of urban vitality is a new technical means and way to study urban vitality. In this paper, points of interest and Weibo check-in geographic markers are used to analyze urban vitality indicators and urban vitality distribution patterns. Through the application of different indexes, the ordinary linear regression and spatial autoregressive models between urban vitality and built environment are established to explore the factors that affect urban vitality. Results of the research show that interest points and social media check-in data can better indicate urban vitality. The urban vitality of the Macao Special Administrative Region is mainly affected by the density of land use, buildings, and public transportation.
\end{abstract}

\section{Introduction}

With the profound transformation of urban society, the importance of cities is becoming a hot topic in urban geography, urban planning, urban economy, and urban management. Jacobs believes that the connection between people's activities and places of residence, as well as the diversity of cities, will make the city important [1]. In the view of Montgomery, a lively space should be made available to a variety of crowded activities, so as to attract different groups of people.

The measurement of the importance of cities is considered the focus of related research. Most traditional studies use research methods to study the importance of cities [2]. Specifically, GPS is used to record the number of active residents in the research and measurement community. This survey method can explain the importance of the surrounding area. However, it is found to be defective because of its time-consuming and labor-consuming characteristics and the inability to cover a large area [3]. Therefore, the ordinary linear regression and spatial autoregressive models between urban vitality and built environment are established to construct different indexes, thus revealing the factors that affect urban vitality [4].

In recent years, with the continuous integration of information technology and positioning technology, big data using geographical location labels (such as mobile signals, point of interest, and social media information) can be used to display a large amount of data about human activities, provide methods to identify physical and social locations in cities, and measure the importance of cities. Because of the advantages and rapid technological improvement, mobile signal data are often adopted to highlight the key city activities [5]. Long Ying takes mobile data as a medium of human activities and collects all signals from mobile phone stations to express vitality and establish an urban importance index system. However, mobile signal data also have some shortcomings, such as the concerns of privacy, limited access, and relatively high costs. The focus, with rich semantics, wide distribution, and easy access, has gained wide acceptance in urban research. The information involved includes services that can represent urban priorities, such as leisure, entertainment, hotels, and shopping. One the other hand, urban economic vitality can be measured by the size of 
above-mentioned indexes and the small amount of catering POIs. In contrast to field data, social media feedback includes details on the location and type of events in different regions, which reflect the vitality of the city. Wu et al., in the literature, suggest the use of Weibo check-in information to reveal the potential importance of the city [6]. Overall, the categories of interest points and Weibo check-in locations can expand the criteria for measuring urban vitality. However, different geographical information may increase the vitality of the city, and the spatial patterns represented by different data can be the basis for measuring the vitality of a city. Therefore, it is necessary to deepen the analysis on its similarities and differences.

Clarifying the mechanism of urban vitality is another key point of this paper. The time and place in people's daily life are intertwined with the surrounding environment, while the living environment is a main factor affecting the vitality and importance of the city [7]. It is found that the vitality of the city is closely related to the living environment of the city, and the land use component is the basic condition of the urban activity area [8]. Different types of land planning will have different effects on the importance of cities, and mixed land use can increase the vitality of cities [9]. MEHTAV is a street full of diversities, which can be characterized by public areas, road facilities, road width, shadows, road traffic, street traffic, architectural features, individual store windows, and geographical features [10]. Therefore, the impact on road vitality cannot be ignored, and buildings are also a key to maintain urban vitality. Ye et al. refer to the method to improve urban vitality with building occupancy spatial indicators and building floor spatial indicators in the literature [5]. Therefore, a wide range of spatial data, such as land use, transport networks, and buildings [11], are used to clarify the impact mechanism of urban environmental indicators including land use, transport networks, and buildings.

With the popularity of intelligent communication devices and the continuous maturity of spatial positioning technology, location-based services (LBSs) have been widely used. The geographical location dimension builds a bridge between the real world and online social network. It aims to share the user's current location and activity information and form a social network based on location service networks (LBSN). Through the movement of the user's location, the sign-in data connect the scattered spatial nodes into a complex network with the track. It resembles a complex network built on geographic space, with transportation networks and road networks. With the popularization of network information technology, social platforms such as Facebook, Twitter, Weibo, and WeChat have produced an endless stream of spatiotemporal data, which contains complex information. This requires rigorous people to explore effective methods to mine these big data. Crowdsourced geographic data processing and mining is a current research hotspot. For example, Wang Bo constructed the public perception sentiment index by using the Weibo check-in data to analyze the spatial and temporal pattern of residents' response to floods. [12] Wang Di used different statistical analysis methods, such as kernel density estimation and focus analysis, to analyze the characteristics of crowd activity from the perspectives of time and space in the urban Weibo attendance data [13]. There must be some close relationship for such an order of magnitude of thousands of nodes and huge components of a complex network between the edges of the nodes. In the entire network, many compact connected block networks are distinguished to divide sub-intervals, which is to divide the corresponding interactive block structure according to the attribute weight. Block structure is an essential property of complex networks. It is formed by the interaction of the elements in the organic system and the relatively stable structure of each element. These relationships include, but are not limited to, the function of the classification structure based on the interaction between the community structure and so on. By mining, we can obtain the fixed subnetwork community structure with high cohesion and low coupling in complex spatial networks. Based on the selection of microblog check-in data mining, this paper will include time attribute data and attribute data in the data and directly reflect the current tourism situation of urban residents. This paper combines a huge amount of data. As a data source of urban block structure mining, it can directly provide a basis for urban spatial structure division through users' dynamic travel. This is of great significance for guiding urban construction and evaluating existing planning schemes.

In this paper, Weibo media check-in data and point of interest (POI) data are utilized to refer to and measure urban vitality and conduct urban planning analysis [14]. Linear regression models and spatial autoregressive models are constructed using land use, urban transportation, buildings, and other built-in environmental factors [15]. It shows the environmental impact mechanism of urban vitality and the stability of the impact mechanism [16]. The study shows that the data scattered in different geographical locations have similar urban spatial patterns [17]. The city's vitality is largely affected by land use, building congestion, and the number of bus stops [18]. This study evaluates the vitality of the current city on the Macao Peninsula [19], discusses the trend of urban space development, determines the mechanism that affects the vitality of the city [20] and the importance of urban vitality to the promotion of urban development, and eventually puts forward targeted policies [21].

\section{Methods}

2.1. Urban Viability Test. To measure urban vitality is one of the important steps to determine the area layout. This paper will determine key indicators based on roads, which will be described by the government or regional planning department according to relevant information [22] to better study the distribution of key areas. In this paper [23], two kinds of geography markup data (POI and social media sign-in data) were used to calculate the space of two kinds of regional viability was calculated, highlight the city vigor, and analyze the space distribution pattern. 
2.1.1. POI-Based Urban Viability Indicators. The point of interest (POI) is the main place where people's daily activities take place, and the POI data have good spatial scale adaptive characteristics. POI data of urban facilities contain information revealing the wide intersections between urban population and the surrounding environment. In this research, the POI data of reviews and Amed maps will be synthesized to calculate the street POI density and the vitality of the Macao Peninsula. The formula is

$$
V_{\text {poi }}=\frac{P_{\text {street }}}{S_{\text {street }}},
$$

where $P_{\text {street }}$ represents the number of all POIs in the region and $S_{\text {street }}$ means the area in the region.

\subsubsection{Indicators of Urban Vitality Based on Social Media} Check-In. Social media check-in information represents a crowd's preference for a particular type of activity or location and can capture some ways of life of people. Here, Sina Weibo's check-in information will be used to calculate nearby social media check-in density information and show the vitality of the city. The formula is

$$
V_{\text {checkin }}=\frac{C_{\text {street }}}{S_{\text {street }}},
$$

where $C_{\text {street }}$ in the formula represents the number of checks in the area and $S_{\text {street }}$ represents the area in the region.

2.1.3. Moran's I. In this paper, Moran's I is used to analyze the autocorrelation of urban importance and indicate the traditional distribution pattern of spatial importance. The formula is

$$
\left\{\begin{array}{l}
I=\frac{n}{S_{0}} \frac{\sum_{i=0}^{n} \sum_{j=0}^{n} W_{i, j} Z_{i} Z_{i}}{\sum_{i=0}^{n} Z_{i}^{2}}, \\
S_{0}=\sum_{i=0}^{n} \sum_{j=0}^{n} W_{i, j} .
\end{array}\right.
$$

The formula shows the deviation between the attribute of the element $i$ and its average value and the spatial weight between the elements $i$ and $j . n$ is equal to the sum of elements; it is the aggregation of the weights of all spatial elements. Moran's $I>0$ denotes spatial autocorrelation: the larger the value, the more obvious the spatial correlation; Moran's $I<0$ denotes spatial negative correlation: the smaller the value, the more obvious the spatial dispersing; and Moran's $I=0$ denotes that the space is random.

2.2. Built Environment. The built environment includes urban buildings and artificial sites, and artificial construction is an important factor affecting the main environment of the city. The built environment described in this paper is made up of land use, transportation, and buildings.
2.2.1. Land Use. Based on the spatial data of land use, this paper calculates the ratio of commercial land, residential land, government land, and industrial land. Shannon's entropy is used to describe the degree of mixed land use. The higher the entropy value, the higher the land utilization ratio. Similarly, the lower the entropy value, the lower the amount of relative mixture. The formula is

$$
\text { Entropy }=\sum_{1}^{n} P_{i} \times \operatorname{In}\left(P_{i}\right) \text {. }
$$

This formula shows the proportion of land area of a certain land use type in a specific area of each land type. $n$ is the number of land classification applications.

2.2.2. Traffic Network. The spatial boundaries of traffic networks, such as roads and buses, have a significant impact on the importance of cities. In this paper, traffic network data are used to calculate road congestion, road network congestion, bus station congestion, and other indicators. The curvature of the road extends to the length of the entire road per unit area. Road network constraints refer to the number of routes in each region. Bus parking restrictions are expressed as the number of bus stations in each service area.

2.2.3. Buildings. The spatial composition of buildings is also an important factor useful in the definition of a city. The following parts of the study will introduce two indicators of building probability, namely, building ratio and land use right probability. Among them, the building area rate of buildings is expressed by the formula. As shown in the formula, the probability of land use right is the percentage of the area occupied by the building block.

$$
\begin{aligned}
\mathrm{FSI}_{i} & =\frac{F_{i}}{A_{i}}, \\
\mathrm{GSI}_{i} & =\frac{B_{i}}{A_{i}},
\end{aligned}
$$

where $F_{i}$ is the floor area of all buildings on the street; $B_{i}$ represents the area summary of the buildings on the street; and $A_{i}$ represents the area of the block.

As shown in Table 1, the built environment is measured by 10 factors, including commercial land, industrial land, government land, land use, road network density, road network building ratio, bus station permeability, building area-to-building area ratio, building area, and soil permeability of buildings.

2.3. Linear Regression Model. First, multiple linear regression is carried out to analyze the impact of built environment on urban vitality. Linear regression (also known as quadratic regression) is one of the most widely used models in routing analysis, considering its ability to reveal the linear relationships of objects. The formula is 
TABLE 1: Completion of environmental indicators.

\begin{tabular}{|c|c|c|c|c|c|c|}
\hline Built environment & Indicators & Number of cases & Scope & Average value & Standard deviation & Variance \\
\hline \multirow{5}{*}{ Land use } & Commercial land ratio & 140 & $0 \sim 0.841$ & 0.404 & 0.172 & 0.414 \\
\hline & Residential land ratio & 140 & $0 \sim 0.344$ & 0.112 & 0.086 & 0.294 \\
\hline & Government land use ratio & 140 & $0 \sim 0.250$ & 0.03 & 0.033 & 0.182 \\
\hline & Industrial land ratio & 140 & $0 \sim 0.353$ & 0.082 & 0.08 & 0.283 \\
\hline & Other land use ratio & 140 & $0 \sim 1.00$ & 0.371 & 0.209 & 0.457 \\
\hline \multirow{4}{*}{ Traffic network } & Land mixed use & 140 & $0 \sim 1.489$ & 0.952 & 0.514 & 0.717 \\
\hline & Road network density & 140 & $0 \sim 3.171$ & 0.763 & 0.361 & 0.601 \\
\hline & Network node density & 140 & $0 \sim 99.803$ & 27.33 & 13.876 & 3.725 \\
\hline & Bus site density & 140 & $0 \sim 5.466$ & 1.099 & 1.007 & 1.004 \\
\hline \multirow{3}{*}{ Buildings } & Building volume & 140 & $0 \sim 9.703$ & 2.72 & 1.994 & 1.412 \\
\hline & Density of buildings & 140 & $0 \sim 0.853$ & 0.351 & 0.21 & 0.458 \\
\hline & Construction area & 140 & & & & \\
\hline
\end{tabular}

$$
y=\beta_{0}+\sum_{j=0}^{m} \beta_{j} x_{j}+\varepsilon .
$$

In the above formula, $y$ is the dependent variable; $x_{j}$ is the independent variable; $\beta_{j}$ is the regression coefficient of the corresponding single variable; $M$ represents the sum of the $m$ of the independent variables involved in the control; $\beta_{0}$ is the initial initialization coefficient; and $\varepsilon$ is the error value.

In this research, we will create a roadmap for POI and social media check-in rates, respectively. Both the dependent variables in the table are POIs (Table Model 1 in Table 2), and the density of social media check-in (Table Model 2 in Table 2) indicates urban vitality. Considering the important differences between the two cities, the continuity model is further adopted to prove the importance of establishing the environmental impact.

2.4. Spatial Autoregressive Model. Linear regression analysis does not consider spatial autocorrelation and spatial distribution of built environment. Therefore, in this paper, we will use the spatial model in practice to further clarify the impact of urban importance on the environment. The formula is

$$
\left\{\begin{array}{l}
y=\rho W_{1} y+\beta_{1} X+\mu, \\
\mu=\lambda W_{2} \mu+\varepsilon \\
\varepsilon: N\left(0, \delta^{2} I\right) .
\end{array}\right.
$$

In the formula, $y$ represents the dependent variable; $p$ is the $W_{1} y$ coefficient of the spatial lag term; $x$ is the independent variable; $\beta$ is the regression coefficient of corresponding independent variable; $\mu$ is the error value; $\varepsilon$ is the random error of variance for service mean value $0 ; W_{1}$ and $W_{2}$ in the residual matrix table represent spatial trend and dependent variable, respectively; and $\lambda$ is the coefficient of spatial error setting.

Different settings can be made according to the parameters in the above formula, and three kinds of spatial autoregressive models can be produced, including spatial lag model, spatial error model, and regression coefficient of spatial error term.
(1) When $\rho \neq 0$ and $\lambda=0$, the model is the spatial lag regression model. The spatial lag regression model considers the spatial correlation of dependent variables. The main point of this paper is that the importance of the neighborhood is not only affected by the dynamic elements but also by the surrounding environment. This suggests the necessity to consider the spatial correlation of vitality.

(2) When $\rho=0$ and $\lambda \neq 0$, the model is the space error model. The spatial error model considers the spatial correlation of the fitting error. The result of this paper shows that the block vitality is not only affected by the relevant driving factors but also by the fitting error of the surrounding block vitality.

(3) When $\rho \neq 0$ and $\lambda \neq 0$, the model is the space Doberman model. The Doberman spatial model not only pays attention to the spatial interval of dependent variables and errors but also concentrates on the influence of spatial variation trend of independent variables. The formula is

$$
\left\{\begin{array}{l}
y=\rho W_{1} y+\beta_{1} X+\lambda W_{2} \mu+W_{3} X \beta_{2}+\varepsilon, \\
\varepsilon: N\left(0, \delta^{2} I\right) .
\end{array}\right.
$$

The parameter $W_{3}$ of the equation is equal to (7) and is the spectrum table of the spatial trend of the independent variable; $\beta_{2}$ is the regression coefficient during this period. The choice of spatial autoregressive model depends on the application. In this paper, the spatial autocorrelation of dependent variable regions is considered with two main indexes for Moran's $I$ test, as shown in Table 3. The results show that both spatial importance variables are in autocorrelation. Moreover, Moran's I value of the spatial regression residuals of POI density and check-in density calculated by GeoDa software is 0.000 . This result represents the saliency test of the two spatial regression residuals. Therefore, this paper chooses the spatial lag regression model to analyze the relationship between the two dynamic indexes and the built environment factors. Two models are also constructed in spatial autoregressive analysis. The dependent variables are still normalized POI density (Model 3 in Table 4) and check-in density (Model 4 in Table 4). The 
TABLE 2: Results of linear regression analysis.

\begin{tabular}{|c|c|c|c|c|c|c|c|c|}
\hline \multirow[b]{2}{*}{ Project } & \multicolumn{4}{|c|}{ Model 1: POI density } & \multicolumn{4}{|c|}{ Model 2: Social media check-in density } \\
\hline & Coefficient & $t$ statistics & $\begin{array}{l}\text { Standard } \\
\text { deviation }\end{array}$ & $p$ value & Coefficient & $t$ statistics & $\begin{array}{l}\text { Standard } \\
\text { deviation }\end{array}$ & $p$ value \\
\hline Constants & -0.278 & $-3.106 * * *$ & 0.090 & 0.002 & 0.066 & 0.629 & 0.104 & 0.531 \\
\hline Building volume & -0.201 & -1.513 & 0.133 & 0.133 & -0.405 & $-2.614 * * *$ & 0.155 & 0.010 \\
\hline Density of buildings & 0.670 & $5.607 * * *$ & 0.119 & $\leq 0.001$ & 0.260 & $1.867 *$ & 0.139 & 0.064 \\
\hline Road network density & 0.436 & $2.940 * * *$ & 0.148 & 0.004 & 0.014 & 0.080 & 0.173 & 0.936 \\
\hline Network node density & 0.023 & 0.197 & 0.115 & 0.844 & 0.240 & $1.797 *$ & 0.134 & 0.075 \\
\hline Bus density & 0.119 & 1.374 & 0.087 & 0.172 & -0.157 & -1.552 & 0.101 & 0.123 \\
\hline Commercial land ratio & 0.273 & $2.902 * * *$ & 0.094 & 0.004 & 0.162 & 1.479 & 0.110 & 0.141 \\
\hline Residential land ratio & 0.038 & 0.394 & 0.095 & 0.694 & 0.241 & $2.172 * *$ & 0.111 & 0.032 \\
\hline $\begin{array}{l}\text { Government land use } \\
\text { ratio }\end{array}$ & 0.004 & 0.033 & 0.118 & 0.974 & 0.097 & 0.707 & 0.137 & 0.481 \\
\hline Industrial land ratio & 0.113 & 1.286 & 0.088 & 0.201 & 0.069 & 0.681 & 0.102 & 0.497 \\
\hline Land mixed use & 0.111 & 1.621 & 0.068 & 0.107 & -0.059 & -0.743 & 0.079 & 0.459 \\
\hline Travel heat & 0.006 & 0.058 & 0.097 & 0.954 & -0.114 & -1.006 & 0.113 & 0.316 \\
\hline$R^{2}$ & 0.613 & & & & 0.212 & & & \\
\hline Adjusted $R^{2}$ & 0.579 & & & & 0.144 & & & \\
\hline AIC & -97.626 & & & & -54.936 & & & \\
\hline SC & -62.326 & & & & -19.637 & & & \\
\hline
\end{tabular}

Note: $* p<0.05, * * p<0.01$, and $* * * p<0.001$ are confidence levels.

TABLE 3: Moran index test results.

\begin{tabular}{lccc}
\hline Vitality indicators & Moran index & $Z$ value & $p$ value \\
\hline POI density & 0.5836 & 11.96 & $\leq 0.001$ \\
Check-in density & 0.5952 & 12.21 & 0 \\
\hline
\end{tabular}

Note: $p<0.001$, confidence levels.

selection of variables in the spatial autoregressive model is based on the linear regression results, that is, the variables eliminated by the stepwise multiple linear regression.

\section{Experiment and Result Analysis}

3.1. Research Area and Experimental Data. The test site of this article is located on the Macao Peninsula. The Macao Peninsula (Portuguese: Macao, English: Macao Peninsula) is one of the three parts that make up Macao, China. It is the main residential area of Macao and the oldest development zone in Macao, with a history of more than 400 years. Macao's historical center is located in the Macao Peninsula, which was listed as the World Cultural Heritage in 2005. The Macao Peninsula was originally an island, but as sediments had accumulated in the upper reaches of the west river, there slowly formed a sandbar facing the north and south, connecting the island to the land to create a peninsula. The Macao Peninsula is composed of five districts: Fathi Ma Tong District, San Andoni Tong District, Lobby District, Wangde Tong District, and Fengshun Tong District (Figure 1). Most of the flat land on the Macao Peninsula is obtained by landfill. The Macao Peninsula covers an area of about 9.1 square kilometers and has a population of about 388,000 .

The Macao Peninsula area is about 9.1 square kilometers, and the population is about 388,000 .

According to the Statistics Bureau of Macao in 2020, Macao has a total population of 682,500 . Among them, most of the Chinese were immigrants from the mainland, accounting for $97 \%$. About 170,000 nonlocal employees are employed in Macao. Macao is a tourist city. Most of the visitors to Macao are mainlanders. Since Macao is dominated by mainlanders and the main social media application in China is Weibo, more than $80 \%$ of Macao people use Weibo. Therefore, the use of Weibo attendance data as social media data in this paper to measure the urban vitality of Macao has a certain universality.

Here, we use geo-tagged data covering the Macao Peninsula, including POI data and social media check-in information. POI data can be obtained from Amap and Dianping data. By the deadline 2019, altogether 40,654 pieces of POI data were collected. POI data include restaurants, businesses, residents, services, schools, and places of entertainment. Each piece of POI data contains information such as latitude, longitude, name, address, and category. Social media login information is captured by API. The valid date was from January 2018 to December 2019. Weibo check-in data have been preprocessed. 145,465 pieces of Weibo check-in data were received. There was a year of difference between POI data and social media, until the return, followed by the entry into a stable period of development. The data on land use and buildings are derived from the Macao Mapping and Cadastral Bureau. Road network data are derived from OpenStreetMap (OSM). The Macao Peninsula experimental area is divided into 140 blocks.

3.2. Dynamic Distribution Model of Macao Peninsula. The spatial distribution patterns of vitality on the Macao Peninsula based on POI data and social media check-in data are shown in Figures 1(b) and 2(b), respectively. Figures 1(a) and 1 (b) respectively shows that the urban spatial vitality indicated by the POI data has a high activity value 
TABLE 4: Spatial autoregressive results.

\begin{tabular}{|c|c|c|c|c|c|c|c|c|}
\hline \multirow{2}{*}{ Project } & \multicolumn{4}{|c|}{ Model 1: POI density } & \multicolumn{4}{|c|}{ Model 2: Social media check-in density } \\
\hline & Coefficient & $Z$ value & $p$ value & Standard deviation & Coefficient & $Z$ value & $p$ value & Standard deviation \\
\hline Spatial lag items & 0.559 & 0.061 & 9.107 & 0.000 & 0.679 & 0.067 & 10.079 & 0.000 \\
\hline Constants & -0.185 & 0.070 & -2.639 & 0.008 & -0.024 & 0.076 & -0.319 & 0.749 \\
\hline Building volume & -0.027 & 0.102 & -0.268 & 0.789 & -0.158 & 0.115 & -1.382 & 0.167 \\
\hline Density of buildings & 0.444 & 0.094 & 4.720 & 0.000 & 0.040 & 0.102 & 0.390 & 0.696 \\
\hline Road network density & 0.419 & 0.114 & 3.662 & 0.000 & -0.159 & 0.126 & -1.256 & 0.209 \\
\hline Network node density & -0.098 & 0.088 & -1.112 & 0.266 & 0.143 & 0.098 & 1.469 & 0.142 \\
\hline Bus density & 0.059 & 0.067 & 0.890 & 0.374 & -0.128 & 0.074 & -1.730 & 0.084 \\
\hline Commercial land ratio & 0.148 & 0.073 & 2.041 & 0.041 & 0.169 & 0.080 & 2.103 & 0.035 \\
\hline Residential land ratio & -0.015 & 0.073 & -0.205 & 0.837 & 0.189 & 0.082 & 2.298 & 0.022 \\
\hline Government land use ratio & -0.025 & 0.090 & -0.280 & 0.779 & 0.111 & 0.100 & 1.104 & 0.269 \\
\hline Industrial land ratio & 0.093 & 0.067 & 1.384 & 0.166 & 0.081 & 0.075 & 1.082 & 0.279 \\
\hline Land mixed use & 0.085 & 0.052 & 1.626 & 0.104 & -0.040 & 0.058 & -0.696 & 0.486 \\
\hline Travel heat & -0.093 & 0.076 & -1.214 & 0.225 & -0.047 & 0.083 & -0.569 & 0.569 \\
\hline $\mathrm{R}^{2}$ & 0.751 & & & & 0.540 & & & \\
\hline AIC & -147.734 & & & & -112.493 & & & \\
\hline SC & -109.493 & & & & -74.251 & & & \\
\hline
\end{tabular}

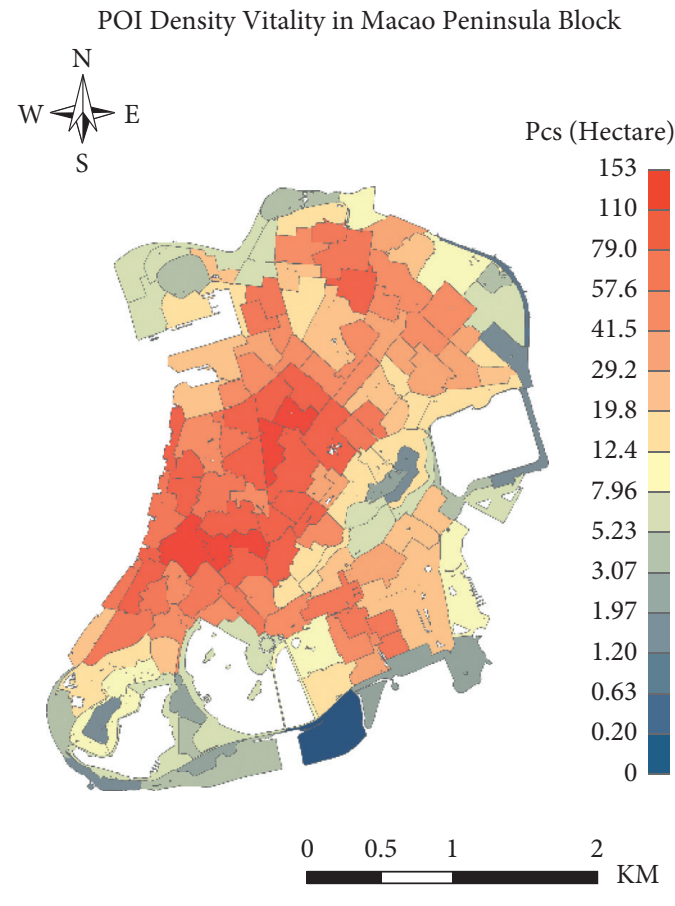

(a)

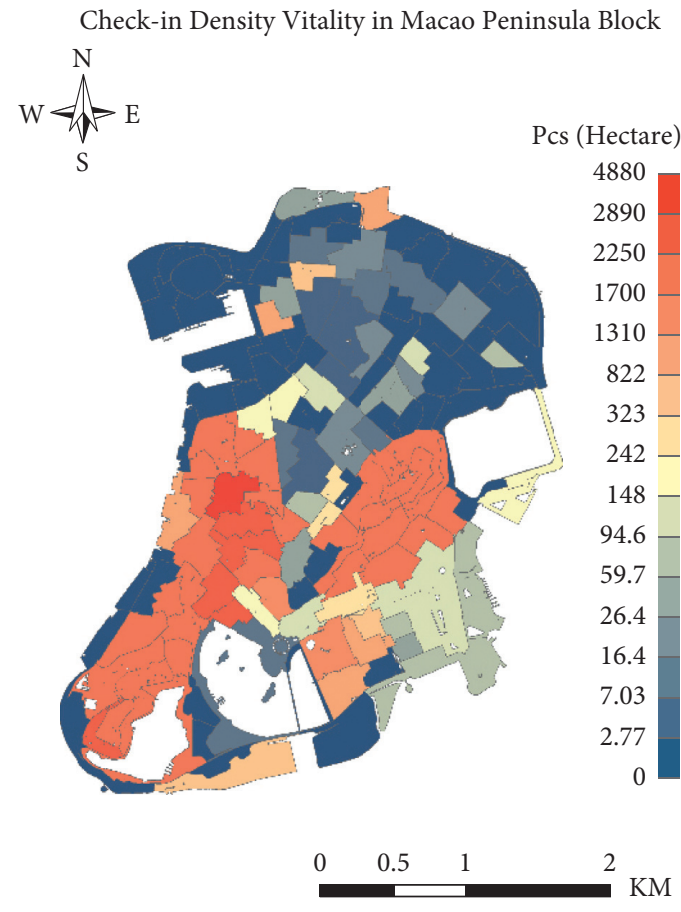

(b)

FIgure 1: Map of POI density patterns in Macao Peninsula block: (a) POI density vitality and (b) check-in density vitality.

aggregation polar core in the mid-west part with a high-high distribution pattern. It suggests that high values surround each other; in the north and southeast parts, the high value is surrounded by the low value. Figures 2(a) and 2(b) show the urban dynamism represented by social media sign-in data. The results show that there are high active value aggregation centers in two historical areas of Macao, which are distributed in the western part and the southwest and the eastern part of the middle Macao Peninsula. The Weibo check-in density pattern map further shows that the urban vitality represented by the sign-in density in the historical city of Macao is highly aggregated. It follows the high-high distribution pattern. 0 represents blocks with less clustering, while 1, 2, and 3 represent groups with confidence levels higher than $0.1,0.04$, and 0.01 , respectively.

The test results show that an obvious imbalance exists in the spatial distribution of urban dynamic areas displayed by social media check-in information and POI data. In other words, high-energy blocks appear in historic blocks, while other areas are depressed. The POI data reveal that its vitality 


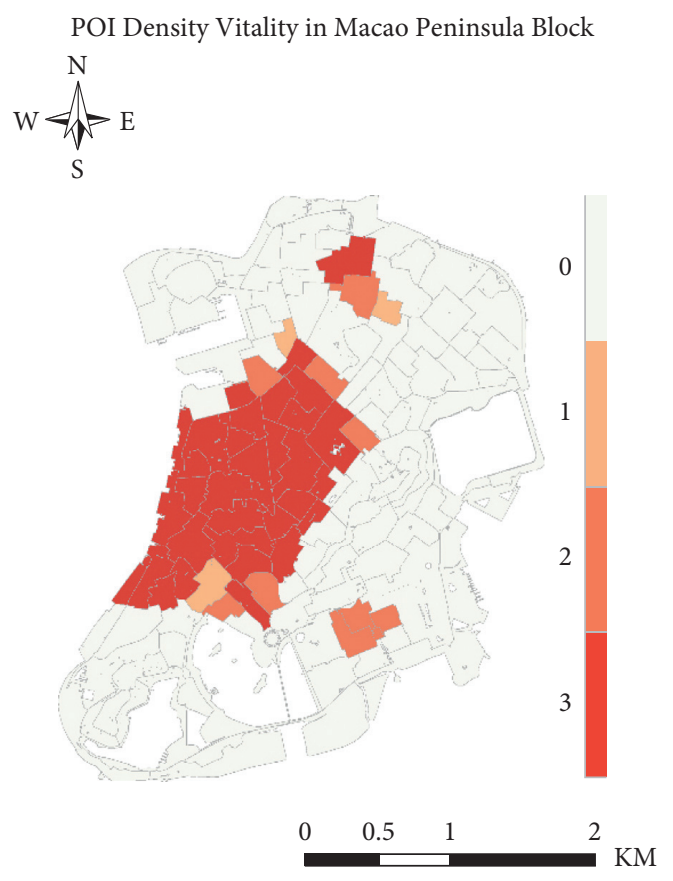

(a)

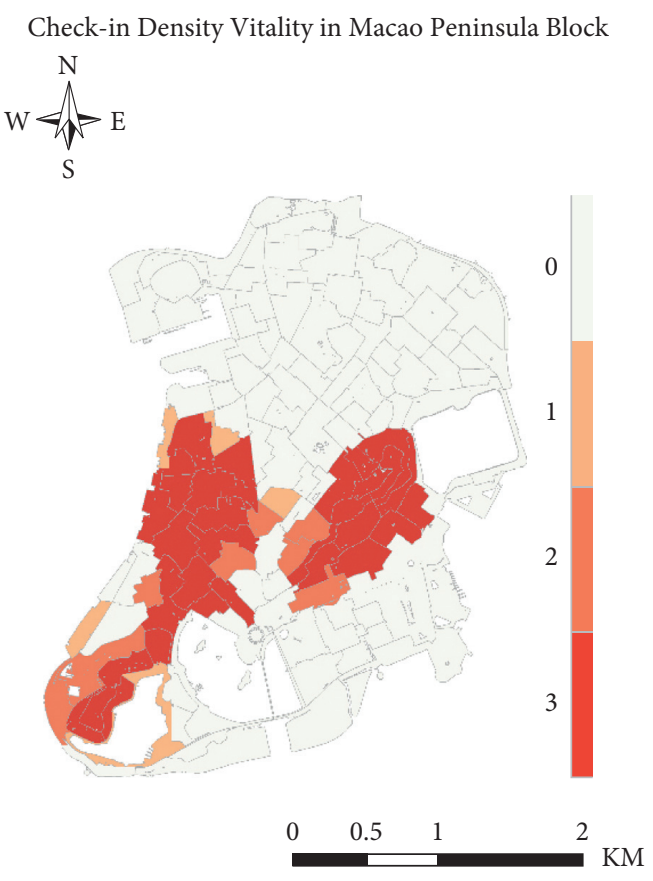

(b)

Figure 2: Map of Weibo sign-in density pattern in Macao Peninsula block: (a) POI density vitality pattern distribution and (b) sign-in density vitality pattern distribution.

is distributed "continuously" in space. It extends from the central and western regions to the north and southeast of the subcenter, and the vitality value of the surrounding area showed a downward trend. The two vitalities also exhibit differences in spatial aggregation. This phenomenon occurs in Macao's historic district, where POI density and vibrant spaces are clustered in the south. It forms a balanced pattern of aggregation.

Furthermore, Figure 1 shows the Moran vitality index of the two cities. Moran's I values of urban importance are 0.574 and 0.586 , respectively, according to POI and social media check-in data. The dynamic regions of the two regions have spatial autocorrelation, both of which are expressed as the dynamic aggregation of the urban dynamic distribution regions. The test of $Z$ and $p$ values shows that the dynamic autocorrelation characteristics of the two urban significance indicators are statistically significant. Therefore, it is suggested that the spatial model of independent analysis of vitality can be used to analyze the specific impact of built environment on urban vitality.

3.3. Linear Regression Analysis. In this paper, we first consider the relationship between independent variables and eliminate the high variance building area of independent variables by collinearity analysis. The final variables classified are the percentage of commercial land, residential land, government land, industrial land, mixed land use, road use, road network use, bus station use, travel heat, building volume ratio, and building occupation density.

The analysis refers to the results of bit multivariate linear geometry in Table 2, which presents parameter coefficients, test $t$ statistics, and $R^{2}$. The two established environmental factors that have the greatest impact on urban vitality are the appearance of buildings and the proportion of commercial land. The results of $R^{2}$ adjustment show that the interpretation rate of urban dynamic environment is $56.8 \%$ and $16.7 \%$, respectively, based on POI and check-in data. As shown by the results of linear regression analysis, the urban vitality of architectural environment interpretation is relatively low.

The observation and experiment demonstrate that the effect of built environment on the vitality of two kinds of cities has common characteristics. Among them, there is an environmental factor with significant effects, that is, the density of building occupation, and the influence coefficient is positive, indicating that this factor generates a positive effect on urban vitality. The influence coefficient is positive, suggesting that this factor has a positive effect on urban vitality. In the future urban construction, increasing the density of buildings and commercial land ratio can enhance the vitality of the city. Among them, the partial correlation coefficient of buildings is the largest, while the buildings with high urban speed have the greatest influence on the importance of promoting the city. According to different data, the impact of the created environment on urban importance varies. For example, the popularity of POI-based public transport stations has significantly increased the importance of cities and has a significant impact on urban vitality. However, the urban vitality based on check-in data has a significant inhibitory effect. In addition, the social activities with high consumption, such as entertainment, shopping, sports, and tourism, are less affected by the density of public transport websites. 
3.4. Spatial Autoregressive Analysis. The influencing factors of urban vitality are mainly obtained by performing stepwise linear regression. Table 4 shows the analysis results of the spatial lag regression model. The $R^{2}$ modes of the two spatial lag modes are $74.2 \%$ and $53.4 \%$, respectively, proving the effectiveness of spatial lag control mode. Compared with the linear model, the interpretation rate of the model increased by $18.3 \%$ and $38.9 \%$, respectively. The results of the lag analysis show that the smaller the values of AIC and SC are, the better the fitting effect of the regression model is. According to linear regression comparison results, the values of AIC and SC decreased by $52.6 \%, 74.9 \%, 114.6 \%$, and $287.3 \%$. It shows that the spatial lag regression model is superior to the linear regression model. Therefore, the spatial lag regression model can better reveal the influence mechanism of abbreviated environment on urban vitality.

The spatial lag regression coefficient expresses the degree of urban vitality affected by its spatial dependence. Among model 3 and model 4 , the coefficients of spatial lag regression terms are 0.559 and 0.679 , respectively. Besides, the significant level is $p \leq 0.001$, which indicates that the regional vitality is greatly affected by the vitality of adjacent regions and all remains positive. On the basis of the sign-in data, the spatial lag regression coefficient of urban vitality is greater than the POI, which indicates that its spatial autocorrelation exerts a great influence on the urban vitality under the signin density indication.

There is no change in the influence of building volume ratio, building occupation density, bus site density, commercial land ratio, industrial land ratio, and mixed land use. It demonstrates that the positive and negative effects of some built-up environmental factors on the importance of cities are frequent. The land density of buildings, the proportion of commercial land, and the ratio of industrial land are always positively related to vitality. Meanwhile, the higher building volume ratio has always had a negative impact on urban vitality.

Compared with the multivariate linear regression model, the influence coefficient of building area rate of the spatial lag regression model is lowered by 0.168 and 0.256 , respectively. Considering the function of domain vitality, the negative effect of building volume ratio is weakened. Similarly, the effect of building occupation density on the two vitalities decreased by 0.226 and 0.220 , respectively. At the same time, the effect of mixed land use on the two vitalities decreased by 0.026 and 0.074 , respectively. As a result, regardless of the dynamic space autocorrelation, the building volume ratio, the density of the building, and the effect of land use on the vitality will be magnified.

\section{Conclusion and Discussion}

Uneven urban development can lead to problems such as unbalanced distribution of facilities and population dispersion, which can contribute to empty cities and heavy traffic. Therefore, it is of great importance to understand the current urban development situation caused by the spatial development of unbalanced areas and to deal with the urban vitality. From the perspective of urban environmental awareness, the present study employs viewpoint (POI) and social media check-in data to create two key indicators and 12 constructive environmental indicators. Furthermore, we adopt the linear regression model (OLS) and spatial autoregressive model to analyze the characteristics of the influence of built environment on urban vitality. In the study of POI density, this phenomenon occurs in the central and western part of the Macao Peninsula with one main and two pairs of patterns. In the activity of check-in density, the experiment is summarized as follows: The urban vitality represented by various data is similar and different in spatial distribution. The vitality of the main areas of the Macao Peninsula is distributed in the central and western regions, while it is weak in the north of, southeast of, and around the island. As shown in the social media records, there is obvious "chaos" in the dynamic distribution of major cities. Besides, the vitality revealed by the POI is "continuous" in space. There also exist major differences between these two types of spatial aggregation. In the POI density space, the phenomenon occurs in the central and western part of the Macao Peninsula with one main and two pairs. In addition, the phenomenon occurs in the historical urban area of Macao in the sign-in density vitality.

The Macao Peninsula is mainly subject to land use and buildings. Among them, commercial land, building area, and bus station density generate significant effects on vitality. The proportion of mixed use of land for construction land has a negative impact. Our goal aims to strengthen the importance of industrial development zones such as Huadi Matang District and its lobby area. Attention should be paid to increasing commercial land, enhancing road network, and improving the visibility of bus stations. In the field of business and traffic, such as the lobby area of the Macao Shopping Centre, its vitality can reduce the proportion of the industrial soil and soil utilization to enhance its vitality. The construction process of some environmental factors affects only part of the key indicators. The intensity of business and road network has a positive effect on POI intensity but had no positive effect on social media audit intensity. In addition, urban planning can adopt different improvement strategies for different aspects of urban vitality.

This study helps to understand the background of urban vitality and formulate policies to improve it. Using POI and social media data to determine the importance of cities can illuminate the relationship between urban importance and many socioeconomic factors including urban dynamics. At the same time, the city's size and utility of focus have a remarkable effect on the spatial distribution of key urban areas. Finally, it is concluded that dense vitality is an important part of spatial analysis and it is necessary to study the problem of plastic space units in order to understand urban vitality.

At present, this paper only uses POI and social media data to determine the importance of cities and clarify the city's importance and the relationship between many social and economic factors, which cover vitality of the city. At the same time, the size and practicality of the city have a great impact on the spatial distribution of the key urban areas. The research needs to be extended to other cities in order to 
study the uniformity of urban vitality distribution and its influencing mechanism. Finally, intensive activity is an important part of spatial analysis. It is necessary to explore plastic spatial units while understanding urban vitality.

\section{Data Availability}

The research data used to support the findings of this study are available from the corresponding author only under license and so cannot be made freely available. Requests for access to these data should be made to the author.

\section{Conflicts of Interest}

There are no potential conflicts of interest in this paper, and all the authors have seen and approved the manuscript to be published.

\section{References}

[1] J. Jacobs, The Death and Life of American cities, Vintage Books, New York, NY, USA, 1961.

[2] P. R. Mass, Towards a Theory of Urban Vitality, Vancouver: University of British Columbia, Vancouver, Canada, 1984.

[3] Y. Liu, X. Liu, and S. Gao, "Social sensing: a new approach to understanding our socioeconomic environments," Annals of the Association of American Geographers, vol. 105, no. 3, pp. 512-530, 2015.

[4] W. Tu, J. Cao, and Y. Yang, "Coupling mobile phone and social media data: a new approach to understanding urban functions and diurnal patterns," International Journal of Geographical Information Science, vol. 31, no. 12, pp. 23312358, 2017.

[5] Y. Ye, D. Li, and X. Liu, "How block density and typology affect urban vitality: an exploratory analysis in Shenzhen, China," Urban Geography, vol. 39, no. 4, pp. 631-652, 2018.

[6] C. Wu, X. Ye, F. Ren, and Q. Du, "Check in behaveiour and spatio-temporal vibrancy:an exploratory analysis in shengzhen,china," Cities, vol. 77, pp. 104-116, 2018.

[7] Y. Yang, Z. Yan, A. Yeh, J. Y. Xie, C. L. Ma, and Q. Q. Li, "Measurements of POI-based mixed use and their relationships with neighbourhood vibrancy," International Journal of Geographical Information Science, vol. 31, no. 4, pp. 658-675, 2017.

[8] W. Tu, R. Cao, Y. Yang et al., "Spatial varitial variations in urban public ridership derived from GPS trajectories and smart card data," Journal of Transport Geography, vol. 69, pp. 45-57, 2018.

[9] J. Lu and F. J Zhang, "Coordinated development of historical and cultural heritage and urban vitality," New Building, no. 1, pp. 32-36, 2016.

[10] Y. Ye and Y. Zhuang, "The hypothesis of spatial pattern evolution and urban vitality: a comprehensive analysis based on road, density and architectural form and functional mixing," International Urban Planning, vol. 32, no. 2, pp. 43-49, 2017.

[11] L. Tang, L. Niu, Y. Xue et al., "Identification and structure extraction of urban road intersection using trajectory big data," Journal of surveying and Mapping, vol. 46, no. 6, pp. 770-779, 2017.

[12] B. Wang, Z. Feng, and H. H. Sun, "Temporal and spatial analysis of urban residents' response to rainstorm and flood based on social media check-in data," Geographical Science, vol. 40, no. 9, pp. 1543-1552, 2020.

[13] D. Wang and X. Zuo, "Analysis of spatial-temporal characteristics of crowd activity based on urban microblog check-in data," Geographical Information World, vol. 27, no. 2, pp. 28-33, 2020.

[14] J. Shan, K. Qin, C. Huang et al., "Discussion on processing and analysis methods of Zhongyuan geographic data," Journal of Wuhan University (Information Science Edition, no. 4, pp. 390-396, 2019.

[15] L. Kong, L. Guan, Y. Ding et al., "Urban regional assessment and its key technologies," Supported by spatial big data Bulletin of Mapping, vol. 8, pp. 100-105, 2017.

[16] D. Ying and Y. Zhou, "Architecture," Urban Theory, vol. 15, pp. 52-62, 2016.

[17] Q. Li, From Gematics to Urban Info Edition), vol. 42, no. 1, pp. 1-6, 2017.

[18] M. Lou, H. Zhang, X. Lei et al., "DBH-tree height model of natural Mongolian broad-leaved mixed forest based on spatial autocorrelation J]," Tree Height Forestry Science, vol. 53, no. 6, pp. 512-530, 2017.

[19] C. Yan, J. Minhe, and T. Song, “The influence of Shanghai City Street on consumption vigor: an Analysis based on POI Diversity and intensity," Journal of Suzhou Institute of Science and Technology (Natural Science Edition), vol. 13, no. 2, pp. 73-80, 2019.

[20] D. Wang, W. Zhong, and D. Xie, "Evaluation and analysis of the built environment in shanghai, such as xie dongcan, Wang de, zhong weijing - exploration of mobile phone signaling data," New Technology Applications, vol. 42, no. 10, pp. 97108, 2018.

[21] S. Wang, Y. Liu, Z. Chen et al., "Methods of urban site scope perception under Dianping data," Journal of Surveying and Mapping, vol. 47, no. 8, pp. 1105-1113, 2018.

[22] Q. Hu, M. Wang, and Q. Li, "Exploring urban hot spots and business circles by using location check-in data," Journal of Mapping, vol. 43, no. 3, pp. 314-321, 2014.

[23] L. Xiang, Z. Chen, J. Wu et al., "Discussion on urban resident population grid method based on optical data and spatial adaptation model," Journal of Geographic Information System, vol. 19, no. 10, pp. 1298-1305, 2017. 